\title{
Salience Signals in the Right Temporoparietal Junction Facilitate Value-Based Decisions
}

\author{
Thorsten Kahnt and Philippe N. Tobler \\ Laboratory for Social and Neural Systems Research, Department of Economics, University of Zurich, 8006 Zürich, Switzerland
}

Value-based decisions optimize the relation of costs and benefits. Costs and benefits confer not only value but also salience, which may influence decision making through attentional mechanisms. However, the computational and neurobiological role of salience in valuebased decisions remains elusive. Here we develop and contrast two formal concepts of salience for value-based choices involving costs and benefits. Specifically, global salience (GS) first integrates costs and benefits and then determines salience based on this overall sum, whereas elemental salience (ES) first determines the salience of costs and benefits before integrating them. We dissociate the behavioral and neural effects of GS and ES from those of value using a value-based decision-making task and fMRI in humans. Specifically, we show that value guides choices and correlates with neural signals in the striatum. In contrast, only ES but not GS impacts decision making by speeding up reaction times. Moreover, activity in the right temporoparietal junction (RTPJ) reflects only ES and correlates with its response-accelerating behavioral effects. Finally, we report an ES-dependent change in functional connectivity between the RTPJ and the locus ceruleus, suggesting noradrenergic processes underlying the response-facilitating effects of ES on decision making. Together, these results support a novel concept of salience in value-based decision making and suggest a computational, anatomical, and neurochemical dissociation of value- and salience-based factors supporting value-based choices.

\section{Introduction}

Every day, individuals make hundreds of value-based choices involving costs and benefits. Costs and benefits do not only determine the value of choice options but also their salience. Although value is used by the organism to guide choices (Montague et al., 2006) and the effects of salience and attention on associative learning have been studied extensively (Mitchell and Le Pelley, 2010), the role of salience in value-based decision making is less clear.

In contrast to the intrinsic properties of a cue (e.g., stimulus intensity), which are sometimes referred to as salience, here we are concerned with acquired salience, i.e., salience that a cue acquires as a result of associative learning. A basic distinction between salience and value is that positive and negative outcomes both are salient but have differently signed values (BrombergMartin et al., 2010; Litt et al., 2011). However, it is unclear how the brain computes salience when choice options are simultaneously associated with costs and benefits (i.e., positive and negative outcomes). For example, assuming that the benefits correspond to the costs, spending $\$ 2.00$ to buy a cookie has the same expected value (EV) as spending $\$ 20.00$ to buy a cake. In

Received July 24, 2012; revised Oct. 22, 2012; accepted Nov. 1, 2012.

Author contributions: T.K. and P.N.T. designed research; T.K. performed research; T.K. analyzed data; T.K. and P.N.T. wrote the paper.

This work was supported by Swiss National Science Foundation Grant PP00P1_128574. We thank the Neuroscience Center Zurich and the Zurich Center for Integrative Human Physiology. We thank J. Fujiwara and C. Burke for help with data acquisition.

Correspondence should be addressed to Thorsten Kahnt, Laboratory for Social and Neural Systems Research, Department of Economics, University of Zurich, Blümlisalpstrasse 10, 8006 Zürich, Switzerland. E-mail: thorsten.kahnt@econ.uzh.ch.

DOI:10.1523/JNEUROSCI.3531-12.2013

Copyright $\odot 2013$ the authors $\quad 0270-6474 / 13 / 330863-07 \$ 15.00 / 0$ such cases, salience could be computed as either the absolute EV [hereafter, global salience (GS)] or, as suggested by a recently published model of attention in learning (Esber and Haselgrove, 2011), the sum of the absolute values of the costs and benefits associated with the decision [hereafter, elemental salience (ES)]. In the above examples, both decisions would have the same level of GS. However, intuitively, the two decisions have different levels of salience, and different levels of attention should be commanded when dealing with them. This intuitive notion is readily captured by ES, which assigns different levels of salience to both decision problems. Nevertheless, it is unclear whether and how either form of salience impacts behavioral and neural decision-making processes. Here we aim to dissociate the two forms of salience and investigate their computational roles in value-based decision making and their underlying neural processes. Specifically, we dissociate EV, GS, and ES using a value-based choice task involving compound offers that entail both costs and benefits.

Previous research has shown that EV is reflected in the firing rate of midbrain dopamine neurons (Tobler et al., 2005; Matsumoto and Hikosaka, 2009) and fMRI activity in the ventral striatum (Knutson et al., 2005; Tobler et al., 2007). Salience and attentional signals have been revealed in a variety of regions, such as the dopaminergic midbrain (Bromberg-Martin et al., 2010), the amygdala (Holland and Gallagher, 1999; Roesch et al., 2010; Li et al., 2011), the lateral prefrontal cortex (Kobayashi et al., 2006), the posterior cingulate cortex (PPC) (Heilbronner et al., 2011), the noradrenergic locus ceruleus (LC) (Aston-Jones and Cohen, 2005), and the ventral attention network (Corbetta and Shulman, 2002), including the right temporoparietal junction (RTPJ). However, it is entirely unknown which of these brain 
A Training (100\% reinforced)
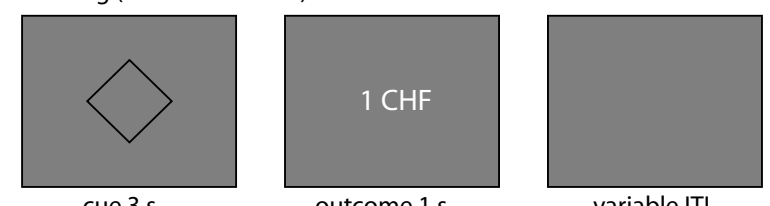

variable ITI

B

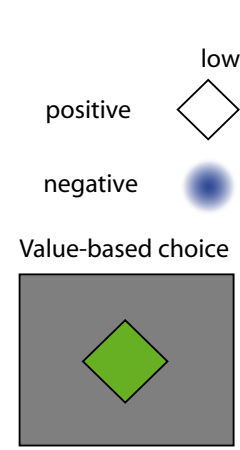
Set I

outcome $1 \mathrm{~s}$

Set II

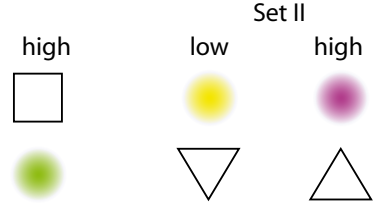

C Value-based choice

cue $3 \mathrm{~s}$

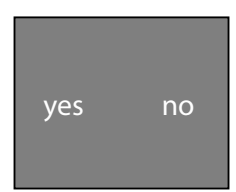

response $1 \mathrm{~s}$

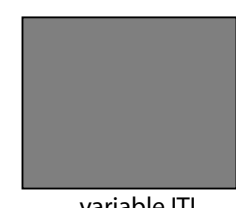

variable ITI
D

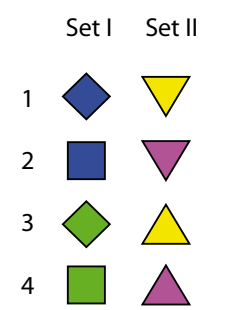

$\begin{array}{cccc}\begin{array}{c}\text { pos/neg } \\ \mathrm{x}_{1} / \mathrm{x}_{2}\end{array} & \begin{array}{c}\text { expected } \\ \text { value (EV) } \\ \mathrm{x}_{1}+\mathrm{x}_{2}\end{array} & \begin{array}{c}\text { global } \\ \text { salience (GS) } \\ \left|\mathrm{x}_{1}+\mathrm{x}_{2}\right|\end{array} & \begin{array}{c}\text { elemental } \\ \text { salience (ES) } \\ \left|\mathrm{x}_{1}\right|+\left|\mathrm{x}_{2}\right|\end{array} \\ 0.1 /-0.1 & 0 & 0 & 0.2 \\ 1 /-0.1 & 0.9 & 0.9 & 1.1 \\ 0.1 /-1 & -0.9 & 0.9 & 1.1 \\ 1 /-1 & 0 & 0 & 2\end{array}$

Figure 1. Stimuli and value-based choice task. $A$, Illustration of a conditioning trial during training. Each cue predicted the outcome with $100 \%$ contingency. $\boldsymbol{B}$, Example association between visual cues and outcomes. Associations were randomized across subjects such that each of the four outcomes was predicted by one color and one shape. C, Illustration of a value-based choice trial. Subjects had to accept or reject the offer that could consist of positive and negative outcomes. Mapping between choice (yes or no) and button response (index or ring finger) was randomized across trials. $\boldsymbol{D}$, Compound offers corresponding to the cue- outcome association in $B$ along with their levels of EV, GS, and ES. ITI, Intertrial interval.

regions are involved in processing (global or elemental) salience during value-based choice.

\section{Materials and Methods}

Subjects. Twenty-one healthy right-handed subjects (nine males; $22.45 \pm$ 0.48 years old, mean \pm SEM) with normal or corrected-to-normal vision were included in the experiment. The study was approved by the Research Ethics Committee of the Canton of Zurich, and subjects provided informed consent to participate.

Stimuli and task. Subjects learned the association between two sets of four visual cues and four possible monetary outcomes $[-1 \mathrm{CHF},-0.1$ CHF, 0.1 CHF, and 1 CHF (Swiss Francs)] using a classical conditioning procedure (Fig. 1A). Each set consisted of two shapes and two colors, such that shapes predicted high and low positive outcomes and colors predicted high and low negative outcomes in one set and vice versa in the other (for an example pairing, see Fig. 1B). This ensured that both colors and shapes were predictive of both costs and benefits, thus controlling for the visual complexity, intensity, and associability of the stimuli. Associations between cues and outcomes were counterbalanced across subjects. In each trial, the cue was presented for $3 \mathrm{~s}$ and was immediately followed by the outcome. Subjects completed 200 training trials in which each cue-outcome pairing was shown 25 times. To test whether they had learned the associations between cues and outcomes, subjects had to rate the value of each cue on a 9-point Likert scale after conditioning.
After training, subjects performed a simple value-based choice task (Fig. 1C) while fMRI data were collected. In each trial, they saw either one of the single cues (single offer) described above or one of eight novel compound cues that had not been presented during training. Compound cues (compound offers) consisted of one shape filled with one color (Fig. $1 D$ ). After stimulus presentation ( $3 \mathrm{~s}$ ), subjects were asked to accept or reject the offer. They indicated their decision by pressing the left (index finger) or right (middle finger) button corresponding to "yes" or "no" on the screen (maximum decision time of $1 \mathrm{~s}$ ). Mappings between "yes" or "no" and buttons were randomized across trials such that motor responses could not be prepared during stimulus presentation. Subjects were told that accepting the offer means that the outcome predicted by the cues will be realized and they will receive or lose the corresponding amount of money, whereas rejecting an offer has no monetary consequences. They were also explicitly informed that both (positive and negative) outcomes would be realized if a compound offer was accepted. Trials were separated by a variable intertrial interval (range of 2-12 $\mathrm{s}$, mean of $4 \mathrm{~s} ; 2 \mathrm{~s}$ fix, plus a variable interval drawn from an exponential distribution with mean of $2 \mathrm{~s}$, truncated at $10 \mathrm{~s}$ ). During each of the eight scanning runs, each single offer and each compound offer was presented three times. Between the decision runs, conditioning was repeated for 32 trials in which each single cue and its corresponding outcome was shown four times in randomized order.

$E V, G S$, and ES. We characterized each decision offer by three variables that are supposed to influence the decision process. The EV of an offer was defined by the sum of the predicted outcomes (EV $=\sum_{i=1}^{n} x_{i}$, where $x$ is the value of a cue $i$ and $n$ is the number of cues of an offer). EV is thought to be directly used to make the choice of whether to accept the offer. The acquired salience of the cues was defined in two ways, as either GS, i.e., the absolute EV (GS $=|\mathrm{EV}|)$, or, as suggested recently (Esber and Haselgrove, 2011), as ES, i.e., the sum of the absolute values of the individual cues (ES $=\sum_{i=1}^{n}\left|x_{i}\right|$ ). Salience is thought to direct cognitive resources, such as attention to the decision, and is thus expected to accelerate choice reaction time (RT). Please note that, within the single offers, GS and ES are identical but orthogonal to EV. However, within the compound offers, EV, GS, and ES are linearly independent (Fig. 1D), and behavioral and neural effects of both forms of salience can be disentangled from each other as well as from the effects of value.

$f M R I$ acquisition and preprocessing. Functional imaging was performed on a Philips Achieva $3 \mathrm{~T}$ whole-body scanner equipped with an eightchannel head coil. During each of the eight test sessions, $216 \mathrm{~T} 2{ }^{\star}$ weighted whole-brain echo planar images ( 37 transversal slices acquired in ascending order) were acquired with a repetition time of $2000 \mathrm{~ms}$. Imaging parameters were as follows: slice thickness, $3 \mathrm{~mm}$; in-plane resolution, $2.75 \times 2.75 \mathrm{~mm}$; echo time, $30 \mathrm{~ms}$; flip angle, $90^{\circ}$. Preprocessing was performed using SPM8 and consisted of slice-time correction, realignment, spatial normalization to the standard echo planar imaging template of the Montreal Neurological Institute, and spatial smoothing using a Gaussian kernel of $8 \mathrm{~mm}$ full-width at half-maximum.

fMRI data analysis. To identify brain regions correlating with EV, GS, and ES, we used a general linear model (GLM) with the following set of regressors for each of the eight fMRI runs: (1) onset of single offers; (2) a parametric regressor of EV ( $z$-scored) of single offers; (3) a parametric regressor of the salience ( $z$-scored) of single offers (note that GS and ES are identical for single offers); (4) onset of compound offers; (5) a parametric regressor of EV ( $z$-scored) of compound offers; (6) a parametric regressor of GS (z-scored) of compound offers; and (7) a parametric regressor of ES ( $z$-scored) of compound offers. $z$ scoring sets the variance of the parametric modulators to one and was performed to allow the direct comparison between the corresponding parameter estimates (e.g., ES vs GS). All regressors were convolved with a canonical hemodynamic response function (HRF) and together with the motion parameters from the realignment procedure regressed against the blood oxygen leveldependent (BOLD) signal in each voxel. Voxelwise second-level $t$ tests were applied to the resulting parameter estimates of the parametric regressors for EV, GS, and ES of compound offers. To identify significant clusters, we used a threshold of $p<0.001$ with an extent threshold of $k>$ 
10 voxels. Correction for multiple comparisons [familywise error (FWE), $p<0.05$ ] was performed at the cluster level. For our a priori region, the ventral striatum, we used small-volume correction (FWE, $p<$ $0.05)$ for $10 \mathrm{~mm}$ spheres around the coordinates $[( \pm 8,8,-8)]$ that are most often reported in combination with "reward" in the NeuroSynth database (Yarkoni et al., 2011).

We tested for a double dissociation of EV and ES coding in the striatum and the RTPJ using regions of interest (ROIs) that were independent of the previous analysis. The RTPJ ROI was created by drawing a sphere $(6 \mathrm{~mm})$ around the coordinates $[(54,-46,23)]$ reported for "attention" in a meta-analysis of RTPJ function (Decety and Lamm, 2007). The ventral striatum ROI was created by drawing spheres $(6 \mathrm{~mm})$ around the coordinates $[( \pm 8,8,-8)]$ defined as described above using the NeuroSynth database.

Functional connectivity analysis. We tested the functional connectivity of the RTPJ using the psycho-physiological interaction (PPI) model (Friston et al., 1997; Kahnt et al., 2009; Park et al., 2011). For each subject, the average time course was extracted from voxels in the RTPJ in which activity significantly ( $p<0.001$, uncorrected) correlated with ES. The time series was multiplied with three indicator variables (for low, middle, and high ES), which were set to 1 for six volumes (12 s) after each onset of each compound-offer trial (with the corresponding ES level) and to 0 otherwise. These three regressors (psycho-physiological regressors) were then included in a GLM along with three HRF-convolved onset regressors for different levels of ES (psychological regressors), the average time course in the RTPJ (physiological regressor), and the six head movement realignment parameters. The parameter estimates of the three psychophysiological regressors reflect the correlation between activity in the RTPJ and activity in every other voxel in trials with different levels of ES. Please note that, because the psycho-physiological regressors were entered into one GLM along with the psychological and physiological regressors, the resulting psycho-physiological parameter estimates are independent of the psychological and physiological effects.

Individual PPI contrasts were computed at the second level by applying the parameter estimates to an ANOVA with three levels (one for each ES level). To identify voxels in which functional connectivity with the RTPJ changed as a function of ES (PPI), we used a linear contrast across the three ES levels (i.e., $-1,0$, and 1, for low, middle, and high, respectively) and a threshold of $p<0.001$ (uncorrected). Correction for multiple comparisons was performed using small-volume correction (FWE, $p<0.05$ ) for $10 \mathrm{~mm}$ spheres surrounding the average anatomical localization of the human LC [ $-4,-37,-26)$ from Keren et al., 2009].

\section{Results}

\section{Value guides and salience facilitates choices}

First, we analyzed the postconditioning ratings to ensure that subjects had learned the association between the eight single cues and the outcomes. A one-way ANOVA with repeated measures on the rating data revealed a significant main effect of value $\left(F_{(3,60)}=174.91, p<0.001\right.$; Fig. $\left.2 A\right)$ and a significant linear effect of value on ratings $(p<0.001)$. This demonstrates that subjects had learned the association between single cues and outcomes. We next tested whether subjects' decisions to accept or reject the compound offers in the value-based choice task were guided by the EV of the offers. Indeed, the rate of accepting a compound offer differed significantly as a function of the EV (main effect in ANOVA: $F_{(2,40)}=545.31, p<0.001$; Fig. $2 B$ ) with significant differences between all levels of EV $(p<0.05)$.

In a next step, we tested the proposed functional role of salience in allocating attention to the decision and thus whether RT varied as a function of the salience of the compound offers. We directly compared the effects of GS and ES on RT by regressing, for each subject, the RT in compound-offer trials against the $z$-standardized values of EV, GS, and ES simultaneously. Across subjects, this analysis revealed significant negative effects of EV $[\beta=-4.02 \pm 1.28$ (mean $\left.\pm \mathrm{SEM}), t_{(20)}=-3.12, p<0.05\right]$ and $\mathrm{ES}\left(\beta=-4.92 \pm 1.82, t_{(20)}=-2.70, p<0.05\right)$ but not GS $(\beta=$
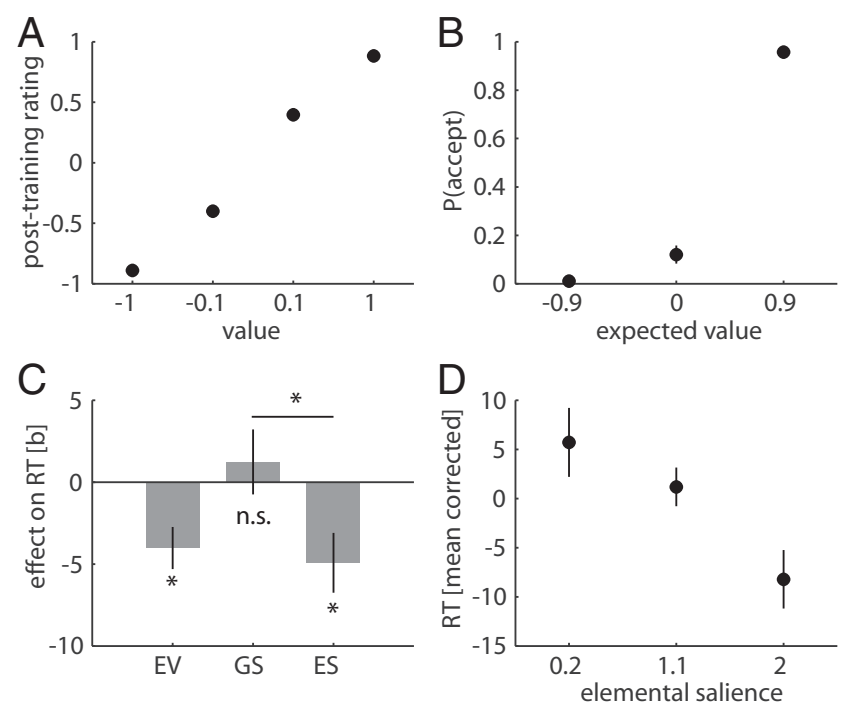

Figure 2. Behavioral effects of EV and salience. $\boldsymbol{A}$, Postconditioning ratings (range -1 to 1 ) as a function of the value of the single cues. Error bars indicating SEM for $n=21$ are smaller than the symbols. $\boldsymbol{B}$, Percentage of accepted offers plotted as a function of the EV of the compound offers. Error bars indicate SEM for $n=21$. C, Effects of EV, GS, and ES on choice RT during compound-offer trials. Bars depict the averaged (across subjects) standardized parameter estimates $(\beta)$ from a within-subject multiple regression of trial-by-trial RT data on EV, GS, and ES. ${ }^{*} p<0.05$. Error bars indicate $S E M n=21$. D, Choice RT in compound trials as a function of ES. Error bars indicate SEM for $n=21$.

$1.34 \pm 1.98, t_{(20)}=0.62, p=0.54$ ) on RT (faster responses with higher ES). Crucially, the negative effect of ES on RT was significantly stronger than the effect of GS on RT $\left(t_{(20)}=-2.50, p<\right.$ 0.05; Fig. 2C). This shows that ES, which is based on the absolute values of the individual outcomes, accounts for RT differences and thus attentional effects during choice. ES had no effect on choice behavior $\left(t_{(20)}=1.19, p=0.25\right)$, demonstrating that it predominantly modulates the speed of value-based choices rather than the value of the offer. This suggests that, in the current experiment, ES directs attention to the decision without modulating its value. Moreover, the findings concur with the intuitive notion that ES is better suited than GS to capture the salience of options with both costs and benefits. However, the effect of salience on RT during single-offer trials was not significant $(\beta=$ $-0.24, p=0.89$ ). This could reflect either a floor effect in RT attributable to the simplicity of choices involving single offers or decreased sensitivity for salience effects in single offers attributable to a smaller range of salience levels.

\section{$\mathrm{EV}$ in the ventral striatum}

With the fMRI data, we first searched for brain areas in which activity increased with the EV in compound-offer trials. In line with our hypothesis, we found significant correlations between $\mathrm{EV}$ and activity in the bilateral ventral striatum [ventral intersection between putamen and caudate; left, $(-12,14,-2), t=4.34$; right, $(12,14,-2), t_{(20)}=4.25, p<0.05$, FWE small-volume corrected; Fig. $3 A, B$ ]. Moreover, significant $(p<0.05$, FWE cluster corrected) correlations were found in the right insula (INS) extending into the lateral orbitofrontal cortex [right BA 47, $(36,17,-8), t_{(20)}=6.47$; Fig. $\left.3 B\right]$, the bilateral intraparietal sulcus (IPS) [left BA $7 / 40,(-39,-49,37), t_{(20)}=5.87$; right BA $7 / 40,(33,-61,43), t_{(20)}=5.59$; Fig. $3 C$ ], and the PCC [BA 23, (6, $\left.-22,28), t_{(20)}=4.95\right]$. To examine whether these regions also signal the EV of single offers, we extracted the parameter estimates for EV in single-offer trials from these regions. Surpris- 

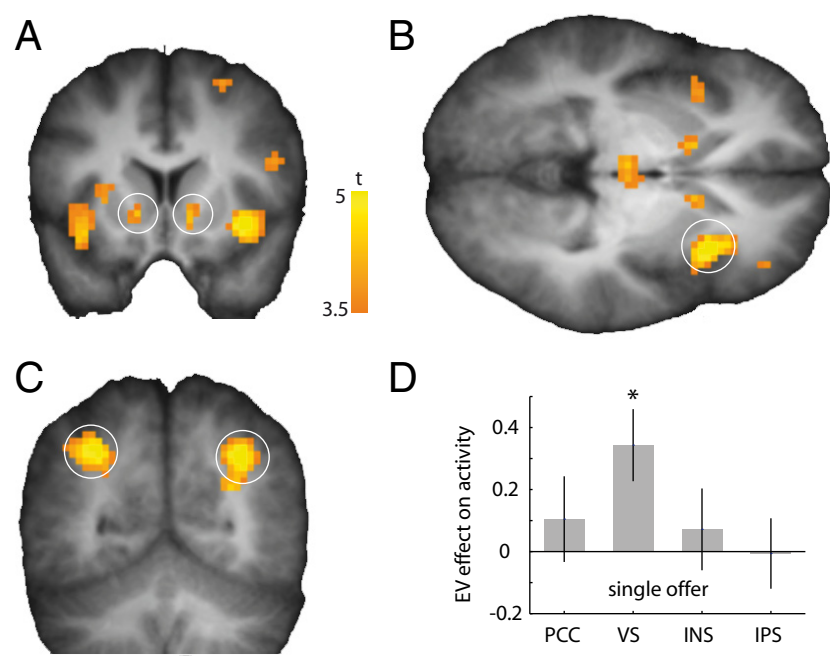

Figure 3. Activity in the ventral striatum correlates with EV. Clusters of voxels in the bilateral ventral striatum (VS) (A), the right anterior INS (B), and bilateral IPS (C) in which fMRI activity correlates with the $\mathrm{EV}$ in compound-offer trials. For illustration, $t$ map from voxelwise onesample $t$ tests on individual parameter estimates of the parametric EV regressors for compound offers are thresholded at $p<0.001$, uncorrected and overlaid on an average normalized anatomical image. $\boldsymbol{D}$, Parameter estimates from the parametric $\mathrm{EV}$ regressors for single offers from regions in which activity correlates significantly (FWE cluster corrected) with the EV in compound-offer trials. ${ }^{*} p<0.05$, Bonferroni corrected. Error bars indicate SEM for $n=21$.

ingly, only activity in the ventral striatum was significantly $\left(t_{(20)}=2.95, p<0.01\right.$, Bonferroni corrected for the number of regions) correlated with the EV on single-offer trials, whereas parameter estimates in the INS, IPS, and PCC were not significant (all $p$ values $>0.46$, uncorrected; Fig. $3 D$ ). This might reflect the fact that integration was necessary to compute the EV of compound offers, whereas the associations between single cues and EV had been learned directly. Thus, the process of online value computation for compound offers might have engaged additional and notably cortical regions.

\section{ES in the RTPJ}

The behavioral analyses above have shown that only ES but not GS influences behavior during value-based choices. Specifically, higher ES was associated with faster RT, suggesting that ES facilitates choices by redirecting attention to the decision. In a next step, we tested whether both variables are also differently expressed in brain activity. Surprisingly, we did not find any significant ( $p<0.05$, FWE cluster corrected) positive correlations with GS. In contrast, ES was significantly $(p<0.05$, FWE cluster corrected) correlated with activity in the RTPJ [BA 22/39, (60, -58, $34), t_{(20)}=4.38$; Fig. $4 A$ ], extending into the superior and middle temporal gyrus. These findings suggest that ES but not GS is represented in brain regions previously associated with orienting attention (Corbetta et al., 2008). Confirming this, a whole-brain comparison between the two types of salience revealed significantly ( $p<0.05$, FWE cluster corrected) higher correlations with fMRI signals for ES compared with GS in the RTPJ $[(60,-58,34)$, $\left.t_{(20)}=7.09\right]$. Moreover, activity in the RTPJ was also correlated with the salience of the single offers $\left(t_{(20)}=2.68, p<0.05\right)$, but it should be noted that the two definitions of salience are identical for the single offers. Moreover, the effects of ES for single and compound offers in the RTPJ were comparable and did not differ significantly $(t=-1.72, p=0.10)$, indicating that, although the range of salience in single offers was smaller than in compound offers, the parametric effects on neural activity are similar.
We further tested the idea that RTPJ activity might mediate the effects of salience on behavior instead of merely correlating with ES. We reasoned that, if activity in the RTPJ would gate the response-facilitating effect of salience, individual differences in the effect of ES on choice RT should be correlated with the effect of ES on RTPJ activity. Indeed, across subjects, we found a significant negative correlation between the behavioral and neural effects of ES ( $r=-0.55, p<0.01$; Fig. $4 B)$. Specifically, in line with our hypothesis, the stronger the effect of ES on RTPJ activity, the stronger the accelerating effect of ES on RT.

\section{Double dissociation of EV and salience coding}

So far, our results suggest that the ventral striatum and the RTPJ are differentially engaged in representing EV and ES. To further confirm this idea, we directly compared the activity in these areas using independent (i.e., unbiased) ROIs (see Materials and Methods). Specifically, we compared the effects of the two variables (EV and ES) across these two brain areas using a $2 \times 2$ (region $\times$ variable) ANOVA with repeated measures on the individual regression coefficients from the parametric regressors. We observed no main effect of ROI $\left(F_{(1,20)}=1.30, p=0.27\right)$ and no main effect of variable $\left(F_{(1,20)}=0.00, p=0.99\right)$ but a significant region $\times$ variable interaction $\left(F_{(1,20)}=24.69, p<0.001\right.$; Fig. $\left.4 C\right)$, indicating differential coding of both variables in both regions. Post hoc tests revealed that EV was significantly more strongly correlated with activity in the ventral striatum than with activity in the RTPJ $(t=2.56, p<0.05)$ and ES was significantly more strongly correlated with activity in the RTPJ than with activity in the ventral striatum $\left(t_{(20)}=3.86, p<0.05\right)$. Further supporting this finding, we also observed a significant region $\times$ variable interaction for the single-cue offers $\left(F_{(1,20)}=26.87, p<0.001\right)$.

\section{RTPJ is functionally connected to the LC}

Noradrenergic projections from the LC have been hypothesized to gate the attention-directing function of RTPJ (Corbetta et al., 2008), but, to our knowledge, empirical evidence for such coupling is lacking so far. We expect enhanced functional connectivity between the RTPJ and the LC in high compared with low ES trials. We tested this hypothesis using a PPI model with the RTPJ as seed region. We found that functional connectivity between the RTPJ and voxels in the putative LC differed significantly as a function of ES $\left[(-6,-37,-23), t_{(40)}=3.61, p<0.05\right.$, FWE small-volume corrected; Fig. $5 A]$. Specifically, functional connectivity between the LC and the RTPJ was stronger during high levels of ES compared with low levels of ES (for illustration, see Fig. $5 B$; please note that absolute connectivity parameter estimates cannot be interpreted). To verify the anatomical location of this cluster around the LC, we plotted it along with a highresolution map of the human LC (Keren et al., 2009). As can be seen in Figure $5 C$, significant voxels were in close vicinity to the anatomical location of the human LC. To further confirm this connectivity result, we performed the connectivity analysis for single offers with high versus low salience and extracted the connectivity estimates in the LC. Indeed, the same saliencedependent change in the functional connectivity between the RTPJ and the LC was found for the single offers $\left(t_{(20)}=2.41, p<\right.$ $0.05)$. Finally, the strength of this salience-dependent connectivity modulation did not differ between single and compound offers $(t=-1.04, p=0.31)$.

\section{Discussion}

In the current study, we formalize and dissociate two concepts of salience, reveal their relative behavioral and neural effects, and 

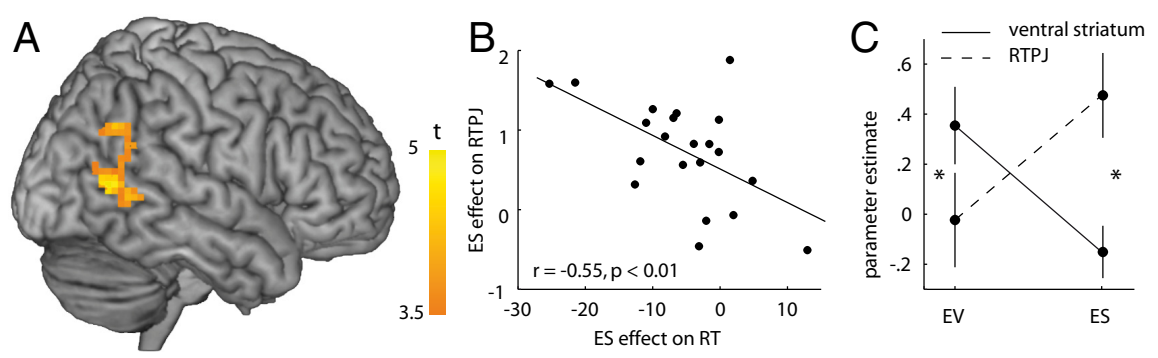

Figure 4. Activity in the RTPJ correlates with the ES of compound offers. A, Surface plot showing a cluster in the RTPJ in which activity correlates with the ES of compound offers. For illustration, activity is thresholded at $p<0.001$ (uncorrected) but survives at $p<0.05$ (FWE cluster correction). B, Significant correlation $(r=-0.55, p<0.01$ ) between the effect of ES on RT and the effect of ES on activity in the RTPJ. The stronger the effect of ES on RT, the stronger the effect of ES on activity in the RTPJ. C, Significant double dissociation between the parametric effects of EV and ES on activity in the ventral striatum and RTPJ, respectively. Data from independent ROIs (see Materials and Methods). Error bars indicate SEM for $n=21$.
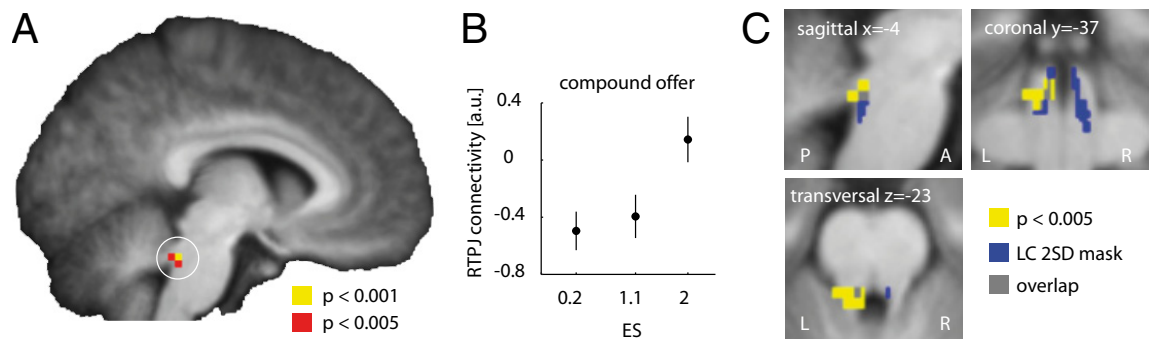

Figure 5. Salience-dependent changes in functional connectivity between RTPJ and LC. $A$, Voxels in the LC in which functional connectivity with the RTPJ is modulated by the ES of compound offers. $t$ map of functional connectivity changes is thresholded at $p<0.001$ (yellow) and $p<0.005$ (red) and overlaid on an average normalized anatomical image. $\boldsymbol{B}$, For illustration, parameter estimates of connectivity in the LC as a function of ES. Error bars indicate SEM for $n=21$. C, Sagittal, coronal, and transversal sections of the functional connectivity $t$ map (yellow) shown in $A$, plotted along with an anatomical 2 SD mask of the human LC (blue) (from Keren et al., 2009).

show that value and salience have dissociable roles in decision making that are paralleled by dissociable neural processes. Specifically, EVs are used to guide choices and were correlated with $B O L D$ responses in the ventral striatum. Conversely, we provide novel evidence that ES but not GS facilitates value-based choice and is correlated with activity in the RTPJ. Moreover, we show that functional connectivity between the RTPJ and the LC is modulated by the ES of the decision, suggesting that noradrenergic mechanisms are involved in salience-dependent choice facilitation.

Our results suggest a novel account for salience, attention, and noradrenaline in value-based decision making. We addressed the conceptual question whether the salience of decisions involving costs and benefits is computed based on either the overall expected outcome (GS) or the sum of the individual positive and negative outcomes associated with a decision (ES). Interestingly, summing up the salience of the individual positive and negative outcomes associated with a cue is the fundamental assumption of a recent model of associative learning (Esber and Haselgrove, 2011). This model proposes a single attentional mechanism to accommodate evidence that predictiveness and uncertainty of cues increase or maintain the salience of cues during learning, which was thought previously to require two separate and somewhat contradictory mechanisms (Mackintosh, 1975; Pearce and Hall, 1980). Our results can thus be considered as an empirical test for this model assumption. In contrast to GS, ES was correlated with faster choice RT, revealing, to our knowledge for the first time, that ES but not GS is behaviorally relevant during value-based decision making. This enhanced behavioral perfor- mance suggests a close link between ES and attentional processing. Specifically, decision making could benefit from attentional resources allocated to salient choices, independent of their actual computational demands. In our task, although the cognitive requirements were the same for high and low salience trials, subjects chose more quickly in high salience trials (ES). Importantly, our results do not suggest faster RT for high salience decisions in general and independent of choice complexity but that attention will be allocated in proportion to the level of salience, which in turn enhances cognitive processing.

Support for attentional mechanisms of ES was also revealed from the fMRI data. In particular, the ES of compound-cue offers was correlated with activity in the RTPJ, and this effect scaled with the performance-enhancing effects of ES. Specifically, the degree to which RT was enhanced by the ES of an offer correlated with the effect of ES on activity in the RTPJ. This suggests that RTPJ activity is not merely correlating with ES but might be critically involved in allocating processing capacities to salient decisions to facilitate decision making. Our results parallel findings in the attention literature, suggesting that the RTPJ belongs to a ventral attention network (Corbetta and Shulman, 2002; Corbetta et al., 2008) that responds to behaviorally relevant stimuli (Geng and Mangun, 2011) even if they are of low visual salience or unattended (Corbetta et al., 2000; Indovina and Macaluso, 2007). Interestingly, within this network, RTPJ is specialized for reorienting attention toward the behaviorally relevant stimulus (Shulman et al., 2009). Our results suggest that acquired ES in value-based decision making directs attentional resources via similar mechanisms, providing novel evidence for the generality of the role of the RTPJ in salience processing.

We found ES-dependent changes in the functional connectivity between the RTPJ and the putative LC, suggesting that the effects of ES in value-based decision making are mediated by noradrenergic processes. This finding is in accord with the hypothesis that the attention-redirecting effects of RTPJ are gated by the activity of noradrenergic LC neurons (Corbetta et al., 2008). Indeed, administration of propranolol, a blocker of $\beta$-adrenergic receptors, attenuates temporoparietal junction responses to infrequent stimuli (Strange and Dolan, 2007), and tracing studies in monkeys have demonstrated that noradrenergic neurons directly innervate the superior temporal gyrus and inferior parietal cortex (Morrison and Foote, 1986; Foote and Morrison, 1987). Noradrenaline has long been associated with arousal and attention-related increases in behavioral performance. In particular, noradrenergic LC neurons respond to target cues with phasic activity increases (AstonJones et al., 1994), and the magnitude of this response is correlated with task performance (Usher et al., 1999). In theory, phasic LC activity may enhance behavioral performance by increasing the gain in sensory, decision, and motor networks 
(Aston-Jones and Cohen, 2005). More specifically, via its neuromodulatory properties, LC noradrenaline facilitates neural processes that are responsible for mapping stimuli and responses, thereby improving RT (Aston-Jones and Cohen, 2005). This idea is in striking accordance with our functional connectivity finding. Functional connectivity between the LC and the RTPJ is enhanced in highly salient trials, which could enable the latter region to mediate the effects of salience on the speed of behavioral responding.

In our study, EV signals (i.e., integrated costs and benefits) predicted subjects' choices and were correlated with activity in the ventral striatum. This finding is in line with reports of value coding in this region (Knutson et al., 2005; Tobler et al., 2007; Yacubian et al., 2007; Lebreton et al., 2009; Cai et al., 2011; Wunderlich et al., 2012). Moreover, previous research on cost-benefit valuation has shown that activity in the ventral striatum correlates with an integrated value signal (Kable and Glimcher, 2007; Tom et al., 2007; Croxson et al., 2009). These striatal value signals are thought to reflect input from the ventral tegmental area in the midbrain (D'Ardenne et al., 2008; Kahnt et al., 2009), in which dopamine neurons code the predicted reward of stimuli (Tobler et al., 2005; Matsumoto and Hikosaka, 2009; Takahashi et al., 2011). Interestingly, dopamine neurons in the midbrain have been suggested to carry both motivational value and salience signals (BrombergMartin et al., 2010). In sharp contrast, our current findings suggest a double dissociation between value signals in the ventral striatum and salience signals in the RTPJ.

In summary, our data suggest complementary roles for value and salience in decision making that are accompanied by dissociable processes on the computational, anatomical, and neurochemical level. Specifically, value information, conveyed by dopamine and represented in the ventral striatum, could support decision making by guiding choices to maximize reward and minimize punishment. Conversely, ES is represented in the RTPJ and facilitates important decisions by redirecting attention, possibly via noradrenergic projections from the LC. Our findings have important implications for neurobiological models of attention-deficit/hyperactivity disorder because the commonly applied pharmacological treatment, methylphenidate, targets both dopamine and noradrenaline reuptake (Del Campo et al., 2011). Finally, these results extend the scope of neurochemical mechanisms underlying value-based decision making beyond the focus on dopamine.

\section{References}

Aston-Jones G, Cohen JD (2005) An integrative theory of locus coeruleusnorepinephrine function: adaptive gain and optimal performance. Annu Rev Neurosci 28:403-450. CrossRef Medline

Aston-Jones G, Rajkowski J, Kubiak P, Alexinsky T (1994) Locus coeruleus neurons in monkey are selectively activated by attended cues in a vigilance task. J Neurosci 14:4467-4480. Medline

Bromberg-Martin ES, Matsumoto M, Hikosaka O (2010) Dopamine in motivational control: rewarding, aversive, and alerting. Neuron 68:815-834. CrossRef Medline

Cai X, Kim S, Lee D (2011) Heterogeneous coding of temporally discounted values in the dorsal and ventral striatum during intertemporal choice. Neuron 69:170-182. CrossRef Medline

Corbetta M, Shulman GL (2002) Control of goal-directed and stimulusdriven attention in the brain. Nat Rev Neurosci 3:201-215. CrossRef Medline

Corbetta M, Kincade JM, Ollinger JM, McAvoy MP, Shulman GL (2000) Voluntary orienting is dissociated from target detection in human posterior parietal cortex. Nat Neurosci 3:292-297. CrossRef Medline

Corbetta M, Patel G, Shulman GL (2008) The reorienting system of the human brain: from environment to theory of mind. Neuron 58:306324. CrossRef Medline

Croxson PL, Walton ME, O’Reilly JX, Behrens TE, Rushworth MF (2009) Effort-based cost-benefit valuation and the human brain. J Neurosci 29: 4531-4541. CrossRef Medline

D'Ardenne K, McClure SM, Nystrom LE, Cohen JD (2008) BOLD responses reflecting dopaminergic signals in the human ventral tegmental area. Science 319:1264-1267. CrossRef Medline

Decety J, Lamm C (2007) The role of the right temporoparietal junction in social interaction: how low-level computational processes contribute to meta-cognition. Neuroscientist 13:580-593. CrossRef Medline

Del Campo N, Chamberlain SR, Sahakian BJ, Robbins TW (2011) The roles of dopamine and noradrenaline in the pathophysiology and treatment of attention-deficit/hyperactivity disorder. Biol Psychiatry 69:e145-e157. CrossRef Medline

Esber GR, Haselgrove M (2011) Reconciling the influence of predictiveness and uncertainty on stimulus salience: a model of attention in associative learning. Proc Biol Sci 278:2553-2561. CrossRef Medline

Foote SL, Morrison JH (1987) Extrathalamic modulation of cortical function. Annu Rev Neurosci 10:67-95. CrossRef Medline

Friston KJ, Buechel C, Fink GR, Morris J, Rolls E, Dolan RJ (1997) Psychophysiological and modulatory interactions in neuroimaging. Neuroimage 6:218-229. CrossRef Medline

Geng JJ, Mangun GR (2011) Right temporoparietal junction activation by a salient contextual cue facilitates target discrimination. Neuroimage 54: 594-601. CrossRef Medline

Heilbronner SR, Hayden BY, Platt ML (2011) Decision salience signals in posterior cingulate cortex. Front Neurosci 5:55. CrossRef Medline

Holland PC, Gallagher M (1999) Amygdala circuitry in attentional and representational processes. Trends Cogn Sci 3:65-73. CrossRef Medline

Indovina I, Macaluso E (2007) Dissociation of stimulus relevance and saliency factors during shifts of visuospatial attention. Cereb Cortex 17: 1701-1711. CrossRef Medline

Kable JW, Glimcher PW (2007) The neural correlates of subjective value during intertemporal choice. Nat Neurosci 10:1625-1633. CrossRef Medline

Kahnt T, Park SQ, Cohen MX, Beck A, Heinz A, Wrase J (2009) Dorsal striatal-midbrain connectivity in humans predicts how reinforcements are used to guide decisions. J Cogn Neurosci 21:1332-1345. CrossRef Medline

Keren NI, Lozar CT, Harris KC, Morgan PS, Eckert MA (2009) In vivo mapping of the human locus coeruleus. Neuroimage 47:1261-1267. CrossRef Medline

Knutson B, Taylor J, Kaufman M, Peterson R, Glover G (2005) Distributed neural representation of expected value. J Neurosci 25:4806-4812. CrossRef Medline

Kobayashi S, Nomoto K, Watanabe M, Hikosaka O, Schultz W, Sakagami M (2006) Influences of rewarding and aversive outcomes on activity in macaque lateral prefrontal cortex. Neuron 51:861-870. CrossRef Medline

Lebreton M, Jorge S, Michel V, Thirion B, Pessiglione M (2009) An automatic valuation system in the human brain: evidence from functional neuroimaging. Neuron 64:431-439. CrossRef Medline

Li J, Schiller D, Schoenbaum G, Phelps EA, Daw ND (2011) Differential roles of human striatum and amygdala in associative learning. Nat Neurosci 14:1250-1252. CrossRef Medline

Litt A, Plassmann H, Shiv B, Rangel A (2011) Dissociating valuation and saliency signals during decision-making. Cereb Cortex 21:95-102. CrossRef Medline

Mackintosh NJ (1975) Theory of attention: variations in associability of stimuli with reinforcement. Psychol Rev 82:276-298. CrossRef

Matsumoto M, Hikosaka O (2009) Two types of dopamine neuron distinctly convey positive and negative motivational signals. Nature 459: 837-841. CrossRef Medline

Mitchell CJ, Le Pelley ME (2010) Attention and associative learning: from brain to behaviour. Oxford, UK: Oxford UP.

Montague PR, King-Casas B, Cohen JD (2006) Imaging valuation models in human choice. Annu Rev Neurosci 29:417-448. CrossRef Medline

Morrison JH, Foote SL (1986) Noradrenergic and serotoninergic innervation of cortical, thalamic, and tectal visual structures in Old and New World monkeys. J Comp Neurol 243:117-138. CrossRef Medline

Park SQ, Kahnt T, Rieskamp J, Heekeren HR (2011) Neurobiology of value 
integration: when value impacts valuation. J Neurosci 31:9307-9314. CrossRef Medline

Pearce JM, Hall G (1980) A model for Pavlovian learning: variations in the effectiveness of conditioned but not of unconditioned stimuli. Psychol Rev 87:532-552. CrossRef Medline

Roesch MR, Calu DJ, Esber GR, Schoenbaum G (2010) Neural correlates of variations in event processing during learning in basolateral amygdala. J Neurosci 30:2464-2471. CrossRef Medline

Shulman GL, Astafiev SV, Franke D, Pope DL, Snyder AZ, McAvoy MP, Corbetta M (2009) Interaction of stimulus-driven reorienting and expectation in ventral and dorsal frontoparietal and basal ganglia-cortical networks. J Neurosci 29:4392-4407. CrossRef Medline

Strange BA, Dolan RJ (2007) Beta-adrenergic modulation of oddball responses in humans. Behav Brain Funct 3:29. CrossRef Medline

Takahashi YK, Roesch MR, Wilson RC, Toreson K, O’Donnell P, Niv Y, Schoenbaum G (2011) Expectancy-related changes in firing of dopamine neurons depend on orbitofrontal cortex. Nat Neurosci 14:1590-1597. CrossRef Medline

Tobler PN, Fiorillo CD, Schultz W (2005) Adaptive coding of reward value by dopamine neurons. Science 307:1642-1645. CrossRef Medline
Tobler PN, O’Doherty JP, Dolan RJ, Schultz W (2007) Reward value coding distinct from risk attitude-related uncertainty coding in human reward systems. J Neurophysiol 97:1621-1632. CrossRef Medline

Tom SM, Fox CR, Trepel C, Poldrack RA (2007) The neural basis of loss aversion in decision-making under risk. Science 315:515-518. CrossRef Medline

Usher M, Cohen JD, Servan-Schreiber D, Rajkowski J, Aston-Jones G (1999) The role of locus coeruleus in the regulation of cognitive performance. Science 283:549-554. CrossRef Medline

Wunderlich K, Dayan P, Dolan RJ (2012) Mapping value based planning and extensively trained choice in the human brain. Nat Neurosci 15:786791. CrossRef Medline

Yacubian J, Sommer T, Schroeder K, Gläscher J, Kalisch R, Leuenberger B, Braus DF, Büchel C (2007) Gene-gene interaction associated with neural reward sensitivity. Proc Natl Acad Sci U S A 104:8125-8130. CrossRef Medline

Yarkoni T, Poldrack RA, Nichols TE, Van Essen DC, Wager TD (2011) Large-scale automated synthesis of human functional neuroimaging data. Nat Methods 8:665-670. CrossRef Medline 